\title{
My Data, My Choice?! The Difference between Fitness and Stress Data Monitoring on Employees' Perception of Privacy
}

\author{
Fabian Richter \\ Project Group Business \& Information Systems \\ Engineering of the Fraunhofer FIT \\ Fabian.Richter@fit.fraunhofer.de
}

Sören Diel

FIM Research Center, University of Bayreuth, Project Group Business \& Information Systems Engineering of the Fraunhofer FIT $\underline{\text { Soeren.Diel@fim-rc.de }}$

\author{
Niklas Gutheil \\ Project Group Business \& Information Systems \\ Engineering of the Fraunhofer FIT \\ Niklas.Gutheil@fit.fraunhofer.de
}

\author{
Christoph Buck \\ Centre for Future Enterprise, QUT Business School, \\ Queensland University of Technology \\ Christoph.Buck@qut.edu.au
}

\begin{abstract}
Besides the vast distribution in the private sector, employers begin to integrate wearables in occupational health management (OHM). Through the implementation of 'stress' and 'fitness monitoring', organizations are able to invest in employees' health and well-being. While employees' consent is mandatory for the implementation, these, in turn, might perceive monitoring as a risk instead of realizing the benefits going along. By conducting an experimental study, we compare employees' perceived privacy risks/costs $(P R C)$ and benefits (PBE) regarding the two monitoring cases. According to our results, employees interpret their stress data as rather sensitive while rating the PBE of fitness monitoring higher. Further, fair communication practices towards employees plays an essential role in the successful implementation of OHM. The research article provides theoretical and practical implications and sheds light on paths for further research regarding actual use behavior, international aspects, and employers' interests.
\end{abstract}

\section{Introduction}

Integrating wearables in organizations' occupational health management (OHM) constitutes an auspicious opportunity to establish a healthy and well cooperating work culture [1-3]. Wearables, being incorporated into clothing and accessories, are body-worn minicomputers collecting data about daily activities, exercise, and vitality [4]. Thereby, the devices gather physiological, behavioral, or ecological data to provide useful services and information. While wearables are ubiquitously integrated into individuals' everyday life, also organizations implement wearables into the workplace $[5,6]$. In OHM, wearables are partly in the early stages, but they offer the potential to actively promote physiological and psychological health in the workplace through real-time data monitoring and tracking [7]. As a result, employees improve their overall health, fitness, and stress levels, which reduces employees' sick leaves, dependency on institutionalized health settings, and the number of doctor's visits during office hours [3], addressing the interests of the whole organization.

Whereas technical abilities promise a broad application of wearables in the workplace, employees' acceptance and consent play a crucial part in the successful implementation. These, in turn, may lack recognition of the benefits of monitoring their personal health, fitness, or stress level while feeling patronized by the employer. From the employees' perspective, the disclosure of health data harbors risks like misuse for performance analysis, misinterpretation of the employer, and passing on to third parties (e.g., insurance companies) [8]. Therefore, perceived loss of privacy, decreasing respect and dignity, and a lack of trust lead to counterproductive behavior, accompany the implementation of wearables, and strain the relationship between employer and employee [9]. Consequently, privacy constitutes itself as the bottleneck of a broad implementation of wearables in OHM.

Some studies have already investigated legal aspects [10], outcomes [11], design principles [12], and employee adoption [13] of wearables in OHM. In detail, they cover technical possibilities, design propositions, and approaches for the implementation of wearables, acceptance and employees' perception on different single monitoring cases, or general overviews on 
wearables' applications in the occupational context [7, 14, 15]. However, while wearables collect diverse types of data that grasp for a context-specific interpretation, former research lacks examinations regarding the perception of privacy in dependence on the collected data type. For example, while users may perceive the collection of fitness related data like steps made per day as non-critical, they might view the permanent tracking of their pulse to monitor their stress level as deep invasion into their privacy. Despite the significant interest by and relevance for companies, regulatory institutions, and employees to understand which types of data are critical to potential users, insights regarding different perceptions of privacy concerns depending on the collected data type are still scarce. Consequently, we aim to provide new insights regarding the perception of privacy in an occupational environment by answering the following research question:

RQ: How are employees' perceived PRC and PBE impacted by the monitoring cases of stress monitoring and fitness monitoring as well as by the associated data types?

We aim to solve the research question by building on privacy research as our theoretical foundation. Thereby, the APCO (Antecedents - Privacy Concerns Outcomes) model represents the cornerstone to explain the willingness to disclose personal data, elucidating the correlations between privacy concerns, its antecedents, and its outcomes. Furthermore, we integrate insights from research on the privacy calculus, explaining privacy through the juxtaposition of privacy risks/costs (PRC) and privacy benefits (PBE), both from a normative and a behavioral standpoint. To test our developed proposition, we collected data from employees who work in an established research center on information systems (IS) and invited them to participate in a study on wearables in the workplace. From a theoretical standpoint, we expand former research by empirically examining privacy-related aspects of wearables' integration in OHM. Thereby, we provide data on employees' perceived differences in dependence of the monitored data type. We furthermore present practical implications to employers, who can derive insights for OHM programs, and employees, who need to be integrated into the implementation and adoption process.

\section{Conceptual Background}

\subsection{Conceptualization of OHM}

Nowadays, organizational requirements for employees are characterized by rising cognitive, informational, and emotional demands, which result in growing psychological stress and an increase in the number of days of incapacity to work [16]. Studies from industrialized countries in North America, Europe, and Australia estimate that the cost of work-related stress to society ranges from 221 million to 187 billion US dollars [17]. In addition to reducing direct costs due to sickness absence or short- or long-term disability, OHM also indirectly aims to increase overall health and thus have a positive impact on employee performance and efficiency [18]. Therefore, in addition to the traditional occupational health and safety perspectives in terms of ergonomics or physical hazards, psychosocial factors in relation to work organization and work culture as well as the psychological health of individuals are becoming increasingly relevant [19]. Thus, it is not surprising that industrialized countries like Germany, $67 \%$ of the companies offer health promotion programs [20].

$\mathrm{OHM}$ is defined as a generic term, partly used synonymously with the subsumed occupational health promotion [21, 22]. Despite (in many countries) legally required measures like occupational health and safety protection or occupational integration management, comprehensive OHM also includes preventive health measures [23, 24]. In particular, the focus of occupational health is shifting from diagnosis and treatment to a preventive approach in the context of earlier detection, intervention and associated treatment of challenges in the work environment $[25,26]$. While occupational health promotion measures for employers are largely voluntary in nature, behavioral interventions to expand personal health literacy and incorporate healthy behaviors are essential in a work environment, dominated by cognitive, sedentary work $[23,24]$. In the present, measures are carried out electronically, for example, through wearables $[1,6]$.

\subsection{Recording Health-Related Data Through Wearables}

Through rapid technological developments, wearables ubiquitously paved their way into the occupational context [27], where they offer multiple possibilities of recording data. The recorded data could technically enable the monitoring of employees' safety, health, or performance $[1,3,6]$. According to existing literature, high perceived stress over a long period of time leads to worse mental and physical health [28]. Also, individuals' fitness positively impacts workplace health [29]. Therefore, stress monitoring and fitness monitoring represent the core of this study.

Stress monitoring provides employees their stresslevel in intervals throughout a workday [30]. For example, recording employees' pulse, heart rate variability (HRV), and galvanic skin response (GSR) is needed to implement stress monitoring [31]. HRV 
describes the variance in the intervals between heartbeats, where lower variance results in a higher level of stress [30]. GSR concerns the electrodermal activity measured on the skin and is associated with emotional arousal [31]. Stress monitoring can leverage employees' overall well-being and contribute to a well-cooperating work culture of organizations.

Fitness monitoring tracks employees' physical activity (PA), so they can make better choices about their health, positively influencing identity and wellbeing [2]. Amongst others, employers require the recording of GPS data, steps and PA, pulse, and blood pressure serving the monitoring case of fitness [32]. For example, steps and PA can be accurately measured with sensors integrated in wearables, such as accelerometers and gyroscopes, without using complex physiological systems [33]. Further, the recording of GPS data serves the monitoring of the distance walked through the day [34]. Stress and fitness monitoring in OHM hold a vast number of advantages for employers and employees, such as the reduction of sick leaves, the independency on institutionalized health setting or the improvement of the employees' overall state for the establishment of a healthy and well cooperating work culture [1-3]. However, although employees might be aware of the benefits of digital OHM systems, privacy concerns are a significant barrier to their successful use within the work environment [35].

\subsection{APCO and the Privacy Calculus}

Informational privacy plays a pivotal role in technology adoption behavior [36]. To explain human beings' willingness to disclose personal data, Smith et al.'s APCO model integrates the perspectives of antecedents (A), privacy concerns (PC), and outcomes $(\mathrm{O})$, identified in prior research related to privacy [37]. Antecedents that determine privacy concerns include factors such as prior experience, awareness, and individual character traits [37]. Privacy concerns, in turn, influence privacy-related individual decisionmaking [37].

Arguing from a normative perspective, that is, an agent attempts to maximize his or her utility [38], the initial APCO model has been widely used and even extended in later years [39]. Thereby, privacy choices are driven by the privacy calculus, which interprets privacy in "economic terms" [40] and weighs perceived PBE and PRC from information disclosure [37, 41]. Smith et al. define PRC as the perceived potential for loss associated with the release of personal information, whereas PBE constitute the net level of favorable outcomes of the disclosure [42, 43]. According to the normative research stream, privacy concerns associate with privacy-seeking behavior like a lower willingness to disclose personal data [44].

However, recent research on privacy integrated principles from behavioral economics and psychology to provide more accurate explanations for actual observed privacy behavior [45]. For example, decision biases and heuristics, affect, and emotions influence the privacy-related decision process (e.g. [38, 46]). Building on Kahnemann and Tversky's seminal Prospect Theory (PT), previous privacy research integrates a perspective of relativeness into privacy decision processes [47]. In doing so, PT allows for an understanding of privacy decisions with respect to a reference point, treating outcomes above or below the reference point as gains and losses [38]. In the same way, Dinev et al. revised the APCO model, integrated elements of the PT, and identified loss aversion, message framing, and endowment effects to influence privacy decision outcomes [45].

\subsection{Relevant Work and Research Propositions}

To provide an overview on existing research, we conducted a literature review following vom Brocke et al. [48]. The search revealed a broad spectrum of research investigating wearables in the context of OHM. The Boolean search string

"wearable*" AND ("occupational health*" OR "*health management" OR "corporate health" OR "corporate wellness" OR "workplace health") AND ("behavior*" OR "privacy" OR "acceptance")

conducted through Web of Science produced 399 research articles, which we then filtered to 48 suitable articles by title and abstract screening. Some studies have already investigated legal aspects [10], outcomes [11], design principles or approaches [12, 49], and employee acceptance [50-53] of wearables in the occupational setting. Former research provides principles and approaches of the implementation of wearables, limiting research on the technical possibilities and ways of integration. Furthermore, a vast amount of literature reviews and overviews shows the spectrum of possible uses of wearables in the occupational context or healthcare $[7,14,15]$. From the employees' perspective, research mostly considers acceptance [50, 52], affordances or constraints [51], or general perception of employees [53].

Despite both being subclasses of health monitoring, recording stress and fitness data differ in the implementation, the associated data types, and thereby employees' acceptance [8, 32]. Recent articles already discover the impact of the monitoring case on the perceived PRC, for example a higher sensitivity towards stress and detailed health information [54, 55] or a relatively lower sensitivity towards fitness tracking [56] 
in a private context. However, existing research lacks knowledge on employees' perception of privacy on the implementation of wearables in the workplace. Particularly, when it comes to OHM, we identified the need for a comparison between different monitoring cases and data types.

Existing findings in research indicate that there are differences between monitoring cases [27], for example, that private users show lower sensitivity towards fitness data compared to their shopping habits [56] while they show higher sensitivity towards stress monitoring and detailed health information [54, 55]. Further, literature reveals differences in concerns between fitness and stress data [57, 58]. We therefore give our first proposition

P1: The monitoring case of employee monitoring impacts perceived PRC of employees, represented by lower perceived PRC on fitness monitoring than stress monitoring.

Analogue to the aforementioned monitoring cases, existing research provides a comparison between data types relevant to this study, which differ in perceived sensitivity [32, 58]. Based on the findings by Fietkiewicz and Ilhan [32], who discovered the highest sensitivity towards GPS data, followed by blood pressure and pulse, we synthesize our second proposition

P2: The data type impacts perceived PRC of employees leading to highest perceived PRC towards GPS data and lowest perceived PRC towards steps and PA data.

As the counterpart of perceived PRC, the perceived PBE is also impacted by the monitoring case [8, 32]. We propose the PBE of monitoring to be influenced by the monitoring case in the opposite way of PRC and build our third proposition:

P3: The monitoring case of employee monitoring impacts perceived PBE of employees, represented by higher perceived PBE on fitness monitoring than stress monitoring.

Finally, existing literature states that open communication, trust seals and fair information practices mitigate privacy concerns [42]. Former research points out that organizational communication raises trust and therefore lowers perceived PRC and privacy concerns in general $[13,59,60]$. Hence, we propose that the communication of the monitoring cases instead of the required data types towards employees or the workers council lowers privacy concerns towards monitoring practices and therefore derive our fourth proposition:

P4: Clear communication impacts perceived PRC of employees. Therefore, we propose lower perceived PRC towards the monitoring case compared to the associated data types.

\section{Methodology}

\subsection{Research Context and Legal Aspects}

This research article focusses the implementation of wearables from a European OHM standpoint. Therefore, the European legal aspects play an important role and we provide some context information [61, 62]. U.S. regulatory standards differ to standards in Europe by their view of privacy as a right versus a commodity [39]. In this research article, the legal bases in Europe and Germany serve as a guideline on employee monitoring possibilities. In Europe, the General Data Protection Regulation (GDPR) governs personal information processing. The GDPR defines personal information as any information relating to an identified or identifiable natural person [62]. In the context of monitoring, the employees' role applies to this definition, resulting in the law's applicability in our examined context. Furthermore, the possibility of clearly assigning the data to a person enables performance analyses of employees, both for stress and for fitness monitoring. Hence, organizations' intent is not relevant in the regulation, but merely the device's technical possibilities. However, as the GDPR is drafted as a prohibition law subject to authorization, personal information processing is generally prohibited. Among other aspects, the employees' express consent concerned with the data processing constitutes an exception [62]. Finally, the works council has a right of codetermination when employers introduce technical facilities that are objectively suitable for monitoring work performance [61], which is regulated in the German Works Constitution Act. As a result, the employees' consent for the implementation of wearables in OHM becomes mandatory.

\subsection{Study Design and Procedure}

To test our propositions, we collected data through an online experiment among employees of a German research center. Thereby, we randomly assigned the participants into two groups with two different monitoring cases (left: stress monitoring; right: fitness monitoring) (Figure 1). According to the assignment, we handed out one of two questionnaires about the monitoring cases stress and fitness monitoring. Both groups answered questions with the same wordings for the query on employees' perception of privacy while one group's questions referred to stress-related data and the other to fitness-related data. For survey design and data collection, we used the software Qualtrics. 


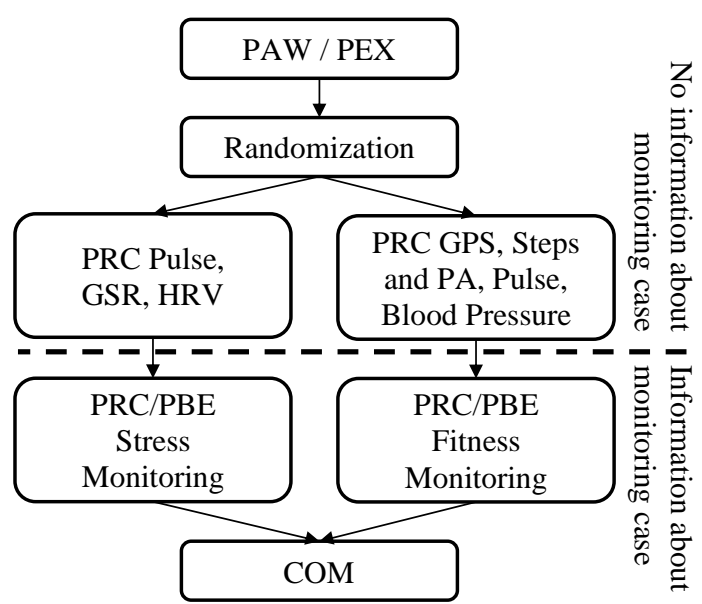

Figure 1: Experimental study design

The pre-test consisted of a representative group of 12 individuals, analyzing the survey regarding usability, ease of use, and correctness of content. After the pretest, we only changed small wording issues. We published the questionnaire in September, the $24^{\text {th }} 2020$ and, after sending reminders for participation in October, the $2^{\text {nd }} 2020$, closed the participation window in October, the $11^{\text {th }} 2020$. We distributed the questionnaire to all 230 employees via the organization's official communication channels (E Mail, Microsoft Teams).

\subsection{Measures}

The constructs in our experiment queried privacy awareness (PAW), privacy experiences (PEX), perceived PRC regarding several types of recorded data and monitoring cases, perceived $\mathrm{PBE}$ of the monitoring cases, and communication preferences (COM). Every item was designed as a 7-point Likert scale, ranging from "I do not agree at all" (1) to "I strongly agree" (7).

We assessed participants' PAW and PEX to ensure that the groups affiliated with the two monitoring cases would show same characteristics towards privacy concerns in general [42]. To assess the perceived PRC and PBE of different monitoring cases and data types, we used items as defined and validated by Krasnova and Veltri [8], Li et al. [63], and Fietkiewicz and Ilhan [32]. Regarding each monitoring case and data type, we asked participants about the PRC in general, perceived risk of their supervisor's misinterpretation of the data, accessibility without knowledge/permission, and concerns regarding the passing on to third parties [8].

We disaggregated the monitoring cases into associated data types, which we identified in section 2.2. We also measured the perceived PRC on those. In sum, we created twelve constructs under study as shown in table 1. It is important to state that we queried the data types first without explaining the intended monitoring case. Later in the questionnaire, we present the monitoring case and query participants' PRC regarding it (see figure 1). Thus, we ensure that participants explicitly rate the data type without being biased by the monitoring case.

\section{Table 1. Constructs}

No. Construct

\begin{tabular}{r|l}
$\mathbf{1}$ & PRC Pulse (Stress) \\
$\mathbf{2}$ & PRC GPS \\
$\mathbf{3}$ & PRC GSR \\
$\mathbf{4}$ & PRC Steps \& PA \\
$\mathbf{5}$ & PRC HRV \\
$\mathbf{6}$ & PRC Pulse (Fitness) \\
$\mathbf{7}$ & PRC Blood Pressure \\
$\mathbf{8}$ & PRC Monitoring Case \\
$\mathbf{9}$ & PBE Monitoring Case \\
$\mathbf{1 0}$ & COM \\
$\mathbf{1 1}$ & PAW \\
$\mathbf{1 2}$ & PEX
\end{tabular}

\subsection{Participants}

Out of the 230 recipients, 173 recipients started the survey, resembling a response rate of $72,2 \%$. The number of responses nearly similarly divides into the previously built groups, i.e., 86 responses for the monitoring case of stress monitoring and 87 responses for fitness monitoring. The deletion of incomplete survey responses resulted in 155 complete responses. We identified careless responses by calculating the standard deviation of each respondents' answers and analyzing the responses for patterns, e.g., 1-2-3-4-5 [64]. Concluding the data screening, we detected no careless responses or significant outliers, leading to a final sample of 155 survey responses. The sample consists of respondents aged between 19 and 54, with a majority of $57,1 \%$ aged 24 to 27 years old and $36,1 \%$ female to $63,9 \%$ male. $64,7 \%$ of respondents specified a university degree as their highest educational qualification, $25,3 \%$ specified university entrance qualification. The average monthly gross income of the respondents is $1821 €$. Concluding, both groups had no significant differences in privacy experiences $(\mathrm{p}=$ $0.797)$ and privacy awareness $(\mathrm{p}=0.881)$.

\section{Results and Discussion}

To examine the impact of different monitoring cases and types of data on employees' perceived PRC and PBE, we collapsed the items into nine constructs. Table 2 shows Cronbach's alpha and the variance inflation factor (VIF) values of the constructs, 
demonstrating no appearance of multicollinearity. Although some constructs fall below the $\alpha$-level of 0.7 , which is considered acceptable, Schmitt [65] states in this respect that a lower reliability is not an obstacle for the use of the factor if it aims at the content coverage of a domain. Since the items are validated by Krasnova and Veltri [8], Li et al. [63], and Fietkiewicz and Ilhan [32], we decided to keep the constructs for the remainder of this article.

Table 2. Composite reliability and variance inflation factors

\begin{tabular}{l|cc}
\multicolumn{1}{l}{ Construct } & $\boldsymbol{\alpha}$ & \multicolumn{1}{c}{ VIF } \\
\hline 1: PRC Pulse (Stress) & 0.718 & 1.279 \\
2: PRC GPS & 0.518 & 1.090 \\
3: PRC GSR & 0.763 & 1.388 \\
4: PRC Steps and PA & 0.510 & 1.076 \\
5: PRC HRV & 0.739 & 1.326 \\
6: PRC Pulse (Fitness) & 0.488 & 1.069 \\
7: PRC Blood Pressure & 0.923 & 2.990 \\
8: PRC Monitoring Case & 0.792 & 1.525 \\
9: PBE Monitoring Case & 0.813 & 2.442
\end{tabular}

\subsection{Perceived PRC (Monitoring Case)}

We measured the impact of the monitoring case on the perceived PRC of employees by conducting a MannWhitney-U-test between two independent samples (two groups). The test results show asymptotic significance $(\mathrm{z}=-3.660 ; \mathrm{p}=0.000)$, indicating a significant difference between the two independent samples. Participants showed lower perceived PRC regarding the monitoring case of fitness monitoring $(\mathrm{M}=4.500 ; \mathrm{SD}=$ 1.095) compared to stress monitoring $(\mathrm{M}=5.250 ; \mathrm{SD}=$ 1.366). Hence, the effect of the monitoring case on perceived PRC was significant $(r=0.294)$. This insight is in harmony with existing literature [8, 32] and confirms our first proposition.

\subsection{Perceived PRC (Data Type)}

The analysis of the effect of different types of data on PRC, conducted through a Friedmann-test (chisquare $=60.164 ; \mathrm{p}=0.000$ ) indicates the exclusion of the null hypothesis. Table 3 shows the results of the post-hoc-test and the mean values of the constructs.

According to existing literature, we proposed an impact of the data type on employees' perceived PRC. Our survey partly confirmed this proposition. While we observed significant differences in PRC between the data types associated with fitness monitoring compared to the data types associated with stress monitoring, we did not observe differences between the interrelated data types. For example, the gap between PRC Pulse (Stress), PRC HRV, and PRC GSR is not significant. We propose that the participants, who are experienced in the field of IS, anticipated the monitoring case based on the compilation of required data types, i.e., imagined the associated monitoring case. Having that in mind, the results are in harmony with the results of PRC monitoring case.

Table 3. Means of constructs and differences between constructs

\begin{tabular}{c|c|cccccc}
\cline { 3 - 8 } \multicolumn{2}{c}{} & \multicolumn{6}{c}{$\mathbf{p}$} \\
$\mathbf{1}$ & 4.934 & $\mathbf{. 0 0 0}$ & n.s. & $\mathbf{. 0 0 1}$ & n.s. & $\mathbf{. 0 0 1}$ & $\mathbf{. 0 0 2}$ \\
$\mathbf{2}$ & 4.072 & & $\mathbf{. 0 0 5}$ & n.s. & $\mathbf{. 0 0 0}$ & n.s. & n.s. \\
$\mathbf{3}$ & 4.890 & & & $\mathbf{. 0 0 8}$ & n.s. & $\mathbf{. 0 1 5}$ & $\mathbf{. 0 1 7}$ \\
$\mathbf{4}$ & 4.118 & & & & $\mathbf{. 0 0 1}$ & n.s. & n.s. \\
$\mathbf{5}$ & 4.912 & & & & & $\mathbf{. 0 0 1}$ & $\mathbf{. 0 0 2}$ \\
$\mathbf{6}$ & 4.110 & & & & & & n.s. \\
$\mathbf{7}$ & 4.131 & & & & & &
\end{tabular}

Finally, the comparison of PRC Pulse (Stress) and PRC Pulse (Fitness) is urgently needed and contributes to a current discussion about privacy risk. We observed a difference between the constructs with a significantly lower value towards the monitoring case of fitness monitoring. This finding is in harmony with the theory by Adjerid et al. [38], who not only considered the objective privacy risk but also the relative perceived privacy risk [38]. Instead of evaluating the PRC of pulse monitoring in an objective manner, the respondents put the date type in context of their relative understanding, resulting in different outputs of perceived PRC. We propose our sample, which is highly educated and experienced towards the field of IS, to strengthen this effect.

\subsection{Perceived PBE (Monitoring Case)}

We also measured the impact of the monitoring case on the perceived PBE of employees through a Mann-Whitney-U-test between two independent samples $(\mathrm{z}=-3.442 ; \mathrm{p}=0.001)$. Regarding the perceived PBE, participants with the monitoring case of fitness monitoring $(M=5.000 ; S D=0.988)$ had higher results than participants with the monitoring case of stress monitoring $(\mathrm{M}=4.250 ; \mathrm{SD}=1.320)$. This results in a significant effect of the monitoring case on perceived PBE of employees $(r=0.276)$. This finding goes well with existing privacy research and furthermore confirms the results of the previous section. Based on the results, we consider an interdependency between PRC and PBE, in terms of a relatively higher PRC negatively impacting perceived PBE of a monitoring case. 


\subsection{Effect of communication}

Participants did not show significant differences in perceived PRC between the monitoring case of stress monitoring and the recording of their pulse $(\mathrm{p}=0.332)$, HRV ( $p=0.3823)$, or GSR $(p=0.072)$. Finally, we did not detect significant differences in perceived PRC between the monitoring case of fitness monitoring and the associated types of data (chi square $=8.159 ; \mathrm{p}=$ 0.086), suggesting that employees do not show deviating perceived PRC between types of data and the associated monitoring case. Nevertheless, participants pointed out that open communication (COM) would lower their privacy concerns $(\mathrm{M}=5.333$; $\mathrm{SD}=1.330)$.

Our fourth proposition was that employees show lower perceived PRC towards the monitoring case compared to the associated data types $[13,59,60]$, as communication lowers the sensitivity. However, it appeared that the effect is not significant. We conclude that, analogously to the other effects, our sample shows high background knowledge and experience in the field of IS, resulting in bias and the ability of seeing coherences. We can exclude the chance of differences between the groups through drawing on the MannWhitney-U-test on PAW and PEX. Nevertheless, the results for COM confirm proposition four. Hence, we propose that there is a possibility of lowering employees' perceived PRC through clear communication, whereas the provision of the monitoring case does not fulfill the desired effect.

\section{Conclusion}

\subsection{Theoretical and Practical Contribution}

This study is a first step to empirically examine the role of privacy in the integration of wearables in OHM. Thereby, we provide data on employee's perceived differences in dependence of the data type that is monitored. Also, our study builds on recent research [8, $32,54-56]$ and is amongst the first to holistically explore differences between the perceived PRC and $\mathrm{PBE}$ of two monitoring cases and the associated data types. Due to the integrated view of the stress- and fitness related data, we expand former studies that have analyzed differences between different monitoring cases [27] and data types [32], while also expanding the customer view on privacy to the occupational context.

Our results have implications for employers, employees, legislators as well as persons in charge of the implementation of OHM in organizations. Building on the legal possibilities, our study compares the perceived PRC and PBE in dependence of different data types. As employees perceived lower PRC and higher
PBE regarding fitness monitoring, we expect a higher chance of employee's acceptance regarding fitness monitoring compared to stress monitoring. However, employers must evaluate the chances of acceptance by their workforce and the works council when planning the implementation of monitoring technology in the workplace. Moreover, we recommend open and fair communication for the implementation of wearables in OHM, for example, through conducting internal surveys and communicating with workers and the workers council. Also, internal workshops where organizations explain the monitoring cases, the need for the relevant data types, the proceeding of personal information and the benefits of wearables in OHM should be integrated in the implementation process. We expect that the involvement of the employee side and the communication of the intent mitigate privacy concerns and raise the acceptance of the workforce.

Furthermore, we present results that are not only relevant to employers' but also add to employees' stakes. Our study shows a mixed reporting towards monitoring in OHM, depending on the intended use. While employees indicate high PBE and low PRC towards fitness monitoring, the results for stress monitoring are contrary. For the successful implementation of wearables in OHM, employees need a holistic overview of possibilities, benefits, and their legal protection. To achieve a well-structured implementation, we recommend the end-to-end integration of all affiliated parties in the process.

Regarding governmental aspects, in Germany the GDPR builds a strong foundation to protect employees comprehensively and strongly towards their privacy while limiting the possibilities of employers. Thereby, organizations' intent is not relevant in the regulation, but merely the device's technical possibilities. Therefore, employers with an intent of raising the occupational well-being are held from the implementation without the acceptance of such measures among their employees.

\subsection{Limitations and Further Research}

Bearing the theoretical contribution and practical implications in mind, it is also important to discuss our study's limitations. First, while this study provides a first step to understand the impact on PRC and PBE in dependence of a certain data type, our sample constitutes employees from one German firm, which is characterized through advanced knowledge in the field of IS. Consequently, further research is necessary both nationally as well as internationally to expand generalizability of the results and to mitigate the risk of misinterpretation due to structural and cultural influences. Additionally, we propose a follow-up study in an industrial setting to mitigate biases through 
knowledge in the field of IS and therefore evaluate differences between the communication data types and monitoring cases. Second, despite research postulating a high correlation between intentions and actual usage of technology [36], further research should investigate actual use behavior, as our study only examined intentions and perceptions. Third, although we integrated numerous types of data, future research might further expand the investigation's scope and investigate differences, for example, considering the device (e.g., smartphone, smartwatch, smart shirts) that is used to track employee data or the technology behind the device (e.g., tracking vs. Tracing technologies, compare discourse of COVID-19 Tracing Applications).

We discovered a possible interdependency between PRC and PBE. In detail, we propose that high PRC leads

\section{References}

[1] Ramani, J.G., S. Madhusudan, A.L. Nila, A. Pradeep, and S. Manibharathi, "IOT Based Employee Health Monitoring System", in 6th International Conference on Advanced Computing and Communication Systems (ICACCS), Coimbatore, Tamil Nadu, India. 2020.

[2] Giddens, L., E. Gonzalez, and D. Leidner, eds., I Track, Therefore I Am: Exploring the Impact of Wearable Fitness Devices on Employee Identity and Well-being, 2016.

[3] Bhattacharjee, P., "Workplace monitoring of employee health using the workstation PC as a platform", in 2014 IEEE Healthcare Innovation Conference (HIC), Piscataway, NJ. 2014.

[4] Baig, M.M. and H. Gholamhosseini, "Smart health monitoring systems: an overview of design and modeling”, J Med Syst, 37(2), 2013.

[5] Singh, T. and A. Johnston, "How Much is Too Much: Employee Monitoring, Surveillance, and Strain”, ICIS 2019 Proceedings, 2019.

[6] Kaur, N. and S.K. Sood, "A Game Theoretic Approach for an IoT-Based Automated Employee Performance Evaluation”, IEEE Systems Journal, 11(3), 2017, pp. 1385-1394.

[7] Khakurel, J., H. Melkas, and J. Porras, “Tapping into the wearable device revolution in the work environment: a systematic review", Information Technology \& People, 31(3), 2018, pp. 791-818.

[8] Krasnova, H. and N.F. Veltri, "Privacy Calculus on Social Networking Sites: Explorative Evidence from Germany and USA", 43rd Hawaii International Conference on System Sciences, Hawaii. 2010.

[9] Posey, C., B.S. Bennett, T. Roberts, and P.B. Lowry, "When Computer Monitoring Backfires: Invasion of Privacy and Organizational Injustice as Precursors to Computer Abuse", Econometrics: Applied to bias towards the perceived PBE of the monitoring case and therefore lower results. Further research should empirically investigate this proposition, for example through an experimental approach. Concluding our research article, we highly recommend to not only shed light on the employees' perspective and center the attention around employers' interests. We discovered lower sensitivity and higher benefits associated with fitness monitoring. However, there is a need to further discuss employers' and employees' interests since they both are responsible for a successful implementation of wearables in the context of OHM.

Econometric Modeling in Microeconomics eJournal, 2011.

[10] Collins, P. and S. Marassi, "Is That Lawful? Data Privacy and Fitness Trackers in the Workplace", International Journal of Comparative Labour Law and Industrial Relations, 2021, pp. 65-94.

[11] Yu, J., J.M. Abraham, B. Dowd, L.F. Higuera, and J.A. Nyman, "Impact of a workplace physical activity tracking program on biometric health outcomes", Preventive medicine, 105, 2017, pp. 135-141.

[12] Yassaee, M., T. Mettler, and R. Winter, "Principles for the design of digital occupational health systems", Information and Organization, 29(2), 2019, pp. 77-90.

[13] Xu, H., H.-H. Teo, B.C.Y. Tan, and R. Agarwal, "The Role of Push-Pull Technology in Privacy Calculus: The Case of Location-Based Services", Journal of Management Information Systems, 26(3), 2010, pp. 135-174.

[14] Khakurel, J., S. Pöysä, and J. Porras, “The Use of Wearable Devices in the Workplace - A Systematic Literature Review", in Smart objects and technologies for social good, O. Gaggi, P. Manzoni, C. Palazzi, A. Bujari, and J.M. Marquez-Barja, Editors. 2017. Springer: Cham.

[15] Lu, L., J. Zhang, Y. Xie, F. Gao, S. Xu, X. Wu, and Z. Ye, "Wearable Health Devices in Health Care: Narrative Systematic Review", JMIR mHealth and uHealth, 8(11), 2020, e18907.

[16] Rothe, I., L. Adolph, B. Beermann, M. Schütte, A. Windel, A. Grewer, U. Lenhardt, J. Michel, B. Thomson, and M. Formazin, Psychische Gesundheit in der Arbeitswelt: Wissenschaftliche Standortbestimmung : Forschung Projekt F 2353, 1st edn., Bundesanstalt für Arbeitsschutz und Arbeitsmedizin (BAuA), Dortmund, Berlin, Dresden, 2017.

[17] Hassard, J., K.R.H. Teoh, G. Visockaite, P. Dewe, and T. Cox, "The cost of work-related stress to society: A 
systematic review", Journal of Occupational Health Psychology, 23(1), 2018, pp. 1-17.

[18] Kirsten, W., "Making the link between health and productivity at the workplace-a global perspective", Industrial health, 48(3), 2010, pp. 251-255.

[19] Beck, D. and U. Lenhardt, "Consideration of psychosocial factors in workplace risk assessments: findings from a company survey in Germany", International Archives of Occupational and Environmental Health, 92(3), 2019, pp. 435-451.

[20] Pronova BKK: Gibt Es In Ihrem Unternehmen Angebote zur Gesundheitsförderung?, https://www.pronovabkk.de/media/downloads/presse studien/studie_bgm_2018/pronovaBKK_BGM_Studie 2018.pdf, accessed 6-13-2021.

[21] Faller, G., "Implementation of Workplace Health Promotion/Workplace Health Management in Germany: State-of-the Art and Need for Further Research", Gesundheitswesen, 80(3), 2018, pp. 278285.

[22] Struhs-Wehr, K., Betriebliches Gesundheitsmanagement und Führung: Gesundheitsorientierte Führung als Erfolgsfaktor im BGM, 1st edn., Springer Fachmedien Wiesbaden, Wiesbaden, 2017.

[23] Scholz, A., U. Singh, A. Ghadiri, and T. Peters, „Nachhaltiges betriebliches Gesundheitsmanagement Empfehlungen für die Implementierung in der Praxis“, in Nachhaltiges Wirtschaften im digitalen Zeitalter, A. Gadatsch, H. Ihne, J. Monhemius, and D. Schreiber, Editors. 2018. GABLER.

[24] Lösch, R., N. Amler, and H. Drexler, “Occupational Safety and Health and Company Integration Management in Germany - A Systematic Review on Implementation of Legal Requirements", Gesundheitswesen, 2021.

[25] Weiner, B.J., M.A. Lewis, and L.A. Linnan, "Using organization theory to understand the determinants of effective implementation of worksite health promotion programs", Health education research, 24(2), 2009, pp. 292-305.

[26] Wang, Y., H. Chen, B. Liu, M. Yang, and Q. Long, “A Systematic Review on the Research Progress and Evolving Trends of Occupational Health and Safety Management: A Bibliometric Analysis of Mapping Knowledge Domains", Frontiers in Public Health, 8, 2020 , p. 81.

[27] Jacobs, J.V., L.J. Hettinger, Y.-H. Huang, S. Jeffries, M.F. Lesch, L.A. Simmons, S.K. Verma, and J.L. Willetts, "Employee acceptance of wearable technology in the workplace", Applied ergonomics, 78, 2019, pp. 148-156.

[28] Epel, E.S., A.D. Crosswell, S.E. Mayer, A.A. Prather, G.M. Slavich, E. Puterman, and W.B. Mendes, "More than a feeling: A unified view of stress measurement for population science", Frontiers in neuroendocrinology, 49, 2018, pp. 146-169.

[29] Blair, S.N., "A Public Health Intervention Model for Work-Site Health Promotion", JAMA, 255(7), 1986, p. 921.

[30] Vaishali, B., S. Amalan, S.P. Preejith, J. Joseph, and M. Sivaprakasam, "HRV based Stress Assessment of Individuals in a Work Environment", in 15th IEEE International Symposium on Medical Measurements and Applications (MeMeA), Bari, Italy. 2020.

[31] Mozgovoy, V., "Stress Pattern Recognition Through Wearable Biosensors in the Workplace: Experimental Longitudinal Study on the Role of Motion Intensity", in 6th Swiss Conference on Data Science (SDS), Bern, Switzerland. 2019.

[32] Fietkiewicz, K. and A. Ilhan, "Fitness Tracking Technologies: Data Privacy Doesn't Matter? The (Un)Concerns of Users, Former Users, and NonUsers", in 53rd Hawaii International Conference on System Sciences. 2020: Hawaii.

[33] Boudet, G., P. Chausse, D. Thivel, S. Rousset, M. Mermillod, J.S. Baker, L.M. Parreira, Y. Esquirol, M. Duclos, and F. Dutheil, "How to Measure Sedentary Behavior at Work?", Frontiers in Public Health, 7 , 2019.

[34] Lidynia, C., P. Brauner, and M. Ziefle, "A Step in the Right Direction - Understanding Privacy Concerns and Perceived Sensitivity of Fitness Trackers", Proceedings of the AHFE 2017 International Conference on Advances in Human Factors and Wearable Technologies. 2018. Springer: Cham, Switzerland.

[35] Yassaee, M. and T. Mettler, "Digital Occupational Health Systems: What Do Employees Think about it?", Information Systems Frontiers, 21(4), 2019, pp. 909-924.

[36] Reith, R., C. Buck, T. Eymann, and B. Lis, "Integrating Privacy Concerns Into the Unified Theory of Acceptance and Use of Technology to Explain the Adoption of Fitness Trackers", International Journal of Innovation and Technology Management, 17(7), 2020.

[37] Smith, H.J., S.J. Milberg, and S.J. Burke, "Information Privacy: Measuring Individuals' Concerns about Organizational Practices”, MIS Quarterly, 20(2), 1996, p. 167.

[38] Adjerid, I., E. Peer, and A. Acquisti, "Beyond the Privacy Paradox: Objective Versus Relative Risk in Privacy Decision Making”, MIS Quarterly, 42(2), 2018, pp. 465-488.

[39] Smith, H.J., "Information Privacy and Marketing: What the U.S. Should (and Shouldn't) Learn from Europe", California Management Review, 43(2), 2001, pp. 8-33. 
[40] Klopfer, P.H. and D.I. Rubenstein, "The Concept Privacy and Its Biological Basis", Journal of Social Issues, 33(3), 1977, pp. 52-65.

[41] Dinev, T. and P. Hart, "An Extended Privacy Calculus Model for E-Commerce Transactions", Information Systems Research, 17(1), 2006, pp. 61-80.

[42] Smith, Dinev, and Xu, "Information Privacy Research: An Interdisciplinary Review”, MIS Quarterly, 35(4), 2011, pp. 989-1015.

[43] Stone, E.F. and L.D. Stone, "Privacy in Organizations: Theoretical issues, Research findings, and Protection mechanisms", Research in Personnel and Human Resources Management, 1990, pp. 349-411.

[44] Malhotra, N.K., S.S. Kim, and J. Agarwal, "Internet Users' Information Privacy Concerns (IUIPC): The Construct, the Scale, and a Causal Model", Information Systems Research, 15(4), 2004, pp. 336355 .

[45] Dinev, T., A.R. McConnell, and H.J. Smith, "Research Commentary-Informing Privacy Research Through Information Systems, Psychology, and Behavioral Economics: Thinking Outside the "APCO" Box", Information Systems Research, 26(4), 2015, pp. 639655.

[46] Acquisti, A., L. Brandimarte, and G. Loewenstein, "Privacy and human behavior in the age of information", Science (New York, N.Y.), 347(6221), 2015, pp. 509-514.

[47] Kahneman, D. and A. Tversky, "Prospect Theory: An Analysis of Decision under Risk", Econometrica, 47(2), 1979, p. 263.

[48] vom Brocke, J., A. Simons, B. Niehaves, K. Riemer, R. Plattfaut, and A. Cleven, "Reconstructing the Giant: On the Importance of Rigour in Documenting the Literature Search Process", in 17th European Conference on Information Systems. 2009: Verona, Italy.

[49] Rodrigues, J.G.P., M. Kaiseler, A. Aguiar, J.P. Silva Cunha, and J. Barros, "A Mobile Sensing Approach to Stress Detection and Memory Activation for Public Bus Drivers", IEEE Transactions on Intelligent Transportation Systems, 16(6), 2015, pp. 3294-3303.

[50] van Acker, B.B., P.D. Conradie, P. Vlerick, and J. Saldien, "Employee acceptability of wearable mental workload monitoring: exploring effects of framing the goal and context in corporate communication", Cognition, Technology \& Work, 2020.

[51] Mettler, T. and J. Wulf, "Physiolytics at the workplace: Affordances and constraints of wearables use from an employee's perspective", Information Systems Journal, 29(1), 2019, pp. 245-273.

[52] Choi, B., S. Hwang, and S. Lee, "What drives construction workers' acceptance of wearable technologies in the workplace?: Indoor localization and wearable health devices for occupational safety and health", Automation in Construction, 84, 2017, pp. 31-41.

[53] Greenfield, R., E. Busink, C.P. Wong, E. Riboli-Sasco, G. Greenfield, A. Majeed, J. Car, and P.A. Wark, "Truck drivers' perceptions on wearable devices and health promotion: a qualitative study", BMC public health, 16, 2016, p. 677.

[54] Peppet, S.R., "Regulating the Internet of Things: First Steps Toward Managing Discrimination, Privacy, Security \& Consent”, Texas Law Review, 93(1), 2014, pp. 85-179.

[55] Nissenbaum, H., "A Contextual Approach to Privacy Online”, Daedalus, 140(4), 2011, pp. 32-48.

[56] Becker, M., A. Kolbeck, C. Matt, and T. Hess, "Understanding the Continuous Use of Fitness Trackers: A Thematic Analysis", in Proceedings of the 21th Pacific Asia Conference on Information Systems. 2017: Langkawi, Malaysia.

[57] Motti, V.G. and K. Caine, "Users' Privacy Concerns About Wearables", in Financial cryptography and data security, M. Brenner, N. Christin, B. Johnson, and K. Rohloff, Editors. 2015. Springer: Heidelberg, New York, Dordrecht, London.

[58] Klasnja, P., S. Consolvo, T. Choudhury, R. Beckwith, and J. Hightower, "Exploring Privacy Concerns about Personal Sensing", in Pervasive Computing. 2009. Springer: Berlin, Heidelberg.

[59] Culnan, M.J. and P.K. Armstrong, "Information Privacy Concerns, Procedural Fairness, and Impersonal Trust: An Empirical Investigation", Organization Science, 10(1), 1999, pp. 104-115.

[60] Andrade, E.B., V. Kaltcheva, and B. Weitz, "SelfDisclosure on the Web: the Impact of Privacy Policy, Reward, and Company Reputation", NA - Advances in Consumer Research, 29, 2002, pp. 350-353.

[61] Arbeitsrecht: Informationen für Arbeitnehmer und Arbeitgeber, Bonn, 2019.

[62] Verordnung (EU) 2016/679. zum Schutz natürlicher Personen bei der Verarbeitung personenbezogener Daten, zum freien Datenverkehr und zur Aufhebung der Richtlinie 95/46/EG (DatenschutzGrundverordnung), 2016.

[63] Li, H., J. Wu, Y. Gao, and Y. Shi, "Examining individuals' adoption of healthcare wearable devices: An empirical study from privacy calculus perspective", International journal of medical informatics, 88, 2016, pp. 8-17.

[64] Meade, A.W. and S.B. Craig, "Identifying careless responses in survey data", Psychological methods, 17(3), 2012, pp. 437-455.

[65] Schmitt, N., "Uses and abuses of coefficient alpha", Psychological Assessment, 8(4), 1996, pp. 350-353. 